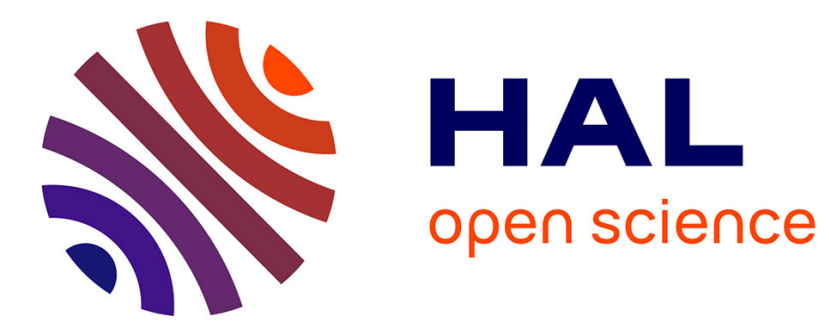

\title{
Experimental impedance assessment of innovative liner under shear grazing flow
}

Fabien Mery, Delphine Sebbane, Rémi Roncen, Estelle Piot, Frank Simon

\section{To cite this version:}

Fabien Mery, Delphine Sebbane, Rémi Roncen, Estelle Piot, Frank Simon. Experimental impedance assessment of innovative liner under shear grazing flow. 25th AIAA/CEAS Aeroacoustics Conference, May 2019, DELFT, Netherlands. 10.2514/6.2019-2517 . hal-02298865v2

\section{HAL Id: hal-02298865 \\ https://hal.science/hal-02298865v2}

Submitted on 2 Jun 2020

HAL is a multi-disciplinary open access archive for the deposit and dissemination of scientific research documents, whether they are published or not. The documents may come from teaching and research institutions in France or abroad, or from public or private research centers.
L'archive ouverte pluridisciplinaire HAL, est destinée au dépôt et à la diffusion de documents scientifiques de niveau recherche, publiés ou non, émanant des établissements d'enseignement et de recherche français ou étrangers, des laboratoires publics ou privés. 


\title{
Experimental impedance assessment of innovative liner under shear grazing flow
}

\author{
F. Méry*, D. Sebbane ${ }^{\dagger}$, R. Roncen ${ }^{\ddagger}$, E. Piot ${ }^{\S}$ and F. Simon \\ ONERA/DMPE - Université de Toulouse, F-31055, Toulouse, France
}

\section{Nomenclature}

$\begin{array}{ll}\phi & \text { Liner perforated sheet hole diameter, }(\mathrm{mm}) \\ \delta & \text { Liner perforated sheet thickness, }(\mathrm{mm}) \\ \text { POA } & \text { Percent Open Area } \\ L_{s} & \text { Thickness of the liner sample } \\ \rho_{0} & \text { Density of the mean flow, }\left(\mathrm{kg} / \mathrm{m}^{3}\right) \\ M_{b} & \text { Bulk flow Mach number } \\ U_{b} & \text { Bulk flow velocity } \\ h & \text { B2A test section height } \\ \omega=2 \pi f & \text { Angular frequency, }(\mathrm{rad} / \mathrm{s}) \\ Z=R+i X \text { Acoustic impedance } \\ \beta & \text { Acoustic reflection coefficient } \\ (x, y, z) & \text { Axial, transversal and vertical coordinates, }(\mathrm{mm}) \\ (U, W) & \text { Axial and vertical mean velocity, }(\mathrm{m} / \mathrm{s}) \\ (u, w) & \text { Axial and vertical acoustic velocity, }(\mathrm{m} / \mathrm{s}) \\ c_{0} & \text { Sound celerity, }(\mathrm{m} / \mathrm{s})\end{array}$

\section{Introduction}

On modern aircrafts, passengers and crew breathe a mixture of fresh and recirculated air. This combination allows the regulation of temperature, pressure and humidity. The air is bled from the engines and supplied to air conditioning units. It is then ducted into the cabin, circulated and eventually drawn into the lower fuselage where it is sucked out by the pressurization outflow valve for the cycle to begin again. Besides creating a safe and comfortable environment, the aircraft air conditioning systems generate noise. The noise radiated from the aircrafts' air conditioning systems is reduced thanks to acoustic liners. These liners present a major design challenge because of the need to address a wide range of conflicting requirements. Acoustic liners must provide high levels of noise reduction over a wide range of operating conditions. They should also be light and flexible to meet strict weight and tight space restrictions.

Until now, acoustic liners for air conditioning systems are made of porous materials, very efficient for sound absorption in the high-frequency range. Locally-reacting liners made of a multiple layers of a honeycomb core topped by a perforated facesheet are classically used in turbofan engine nacelles for mitigating fan noise. ${ }^{1,2}$ These liners are denoted as SDOF (Single Degree of Freedom) or DDOF (Double Degree of Freedom) liners depending on the number of perforate-over-honeycomb layers. Mid-frequency noise attenuation (in the range of $1-5 \mathrm{kHz}$ ) can easily be obtained by tuning the liner acoustic impedance to a target

\footnotetext{
*Research Scientist, fabien.mery@onera.fr

†Technician, delphine.sebbane@onera.fr

${ }^{\ddagger}$ Research scientist, remi.roncen@onera.fr

$\S$ Research scientist, estelle.piot@onera.fr

๑ Research scientist, frank.simon@onera.fr
} 
value specific to the duct environment (geometry, flow Mach number, noise source modal content). ${ }^{3}$ The impedance achieved by a SDOF or DDOF liner depends on its geometry (porosity and holes diameter of the facesheets, honeycomb depth), on the grazing flow features, and on the noise source level. ${ }^{4}$ During the ALIAS project, ${ }^{5}$ a simulation-based design process was implemented to assess the liners concepts that were the best suited for attenuating the air pump noise in the mid-frequency range. A trade-off between acoustic efficiency, weight and cost manufacturing issues was made before selecting the liners.

In the framework of the IDEAS Project funded within the European initiative CleanSky 2 SYSTEMITD, ONERA, the French Aerospace Lab, and the SMEs ATECA and Poly-Shape combine their research and technological capabilities to propose new ideas in the domain of acoustic liners and in-duct modal detection for air conditioning systems from Liebherr Aerospace. A compact innovative acoustic liner is designed in order to mitigate this jet pump noise source over all the frequency range, while meeting the strict weight, costs and tight space restrictions.

This paper presents two concepts of liner for a large frequency range with high industrial constraints. These liner architectures need to be assessed regarding the flow effect and the incident sound pressure level. The acoustic behavior and the effect of a grazing flow on the impedance of each liner concept are assessed in this paper. This liner concept analysis enables to increase our experimental data base and to identify reliable liner models to optimize the best liner solution regarding industrial cases.

\section{Description of the experiments and impedance eduction methodologies}

\section{II.A. Industrial context}

The jet pump is composed of an inlet, a mixing part containing the jet and a diffuser part (see on figure 2). The jet flow enables to entrain a secondary flow.

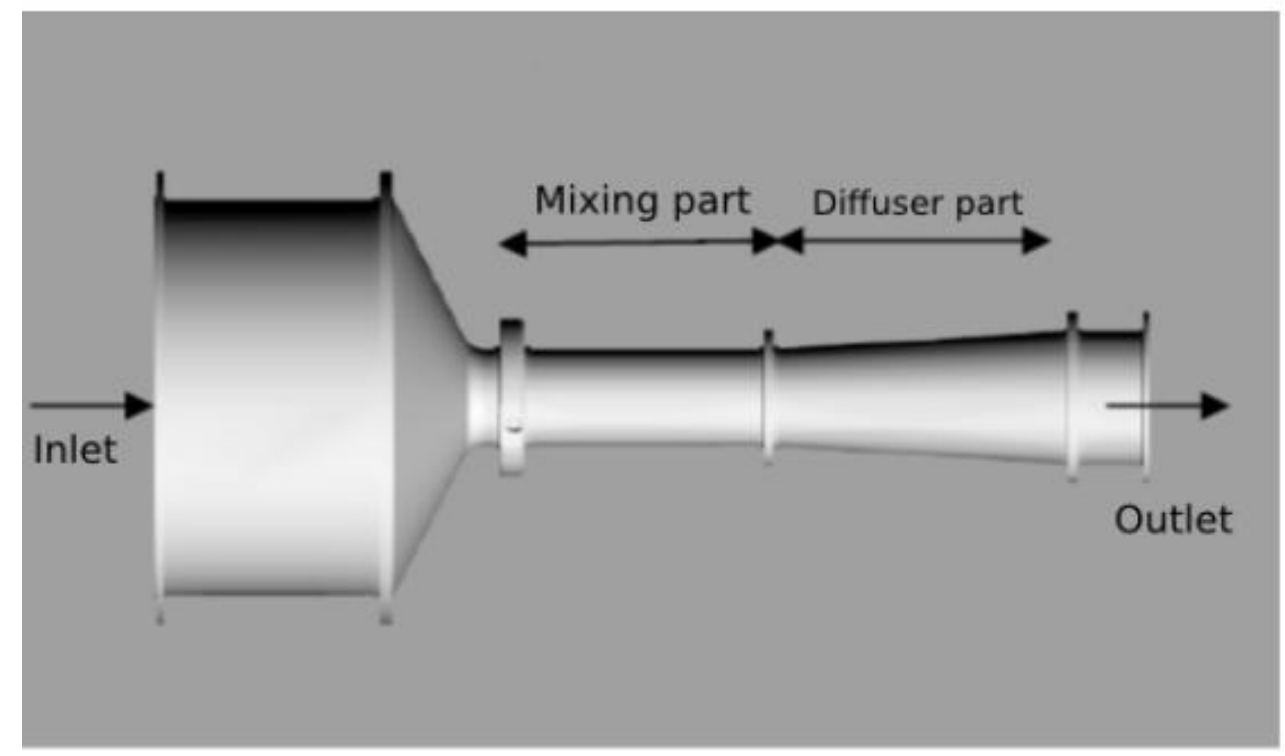

Figure 1. Sketch of the Jet Pump

The interesting frequencies for this problem range from $200 \mathrm{~Hz}$ up to $5000 \mathrm{~Hz}$. The measured spectra out of this jet pump exhibit high sound pressure levels in all this frequency range. The noise induced by this jet pump is more likely to be broadband noise with no preferred frequency. The liners design process that has been performed during the IDEAS project takes into account the real shear flow for several typical flow configurations. The target impedance that must be achieved by the liner architectures is optimised with ONERA's optimisation platform OPAL. The present paper deals with the last part of OPAL platform: experimental validation. This part is important to enhance the confidence level on the optimization results. Dealing with new liner concepts will enable to boost the efficiency of the platform. 


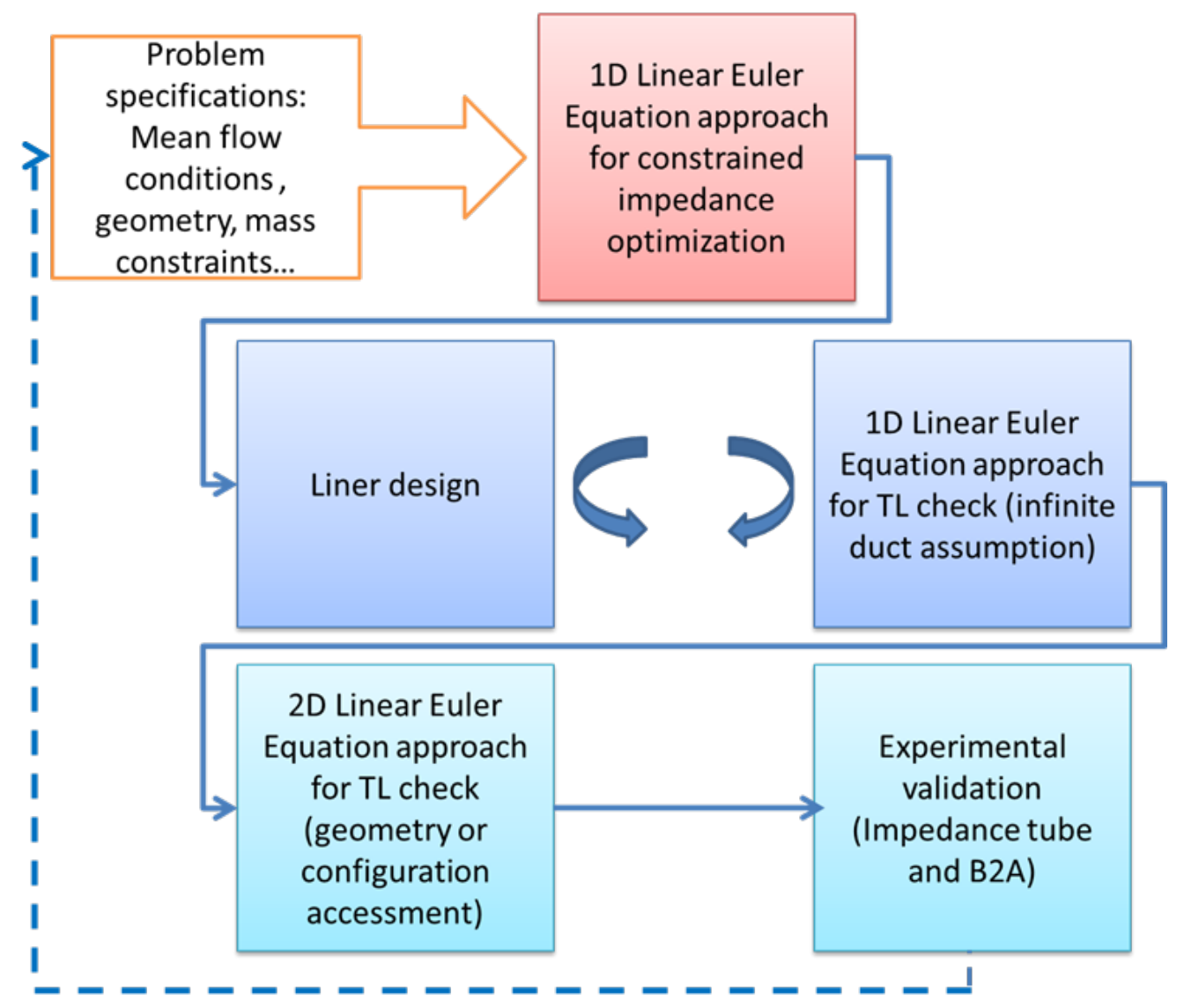

Figure 2. OPAL platform principle 


\section{II.B. Tested liners: samples definition}

For each liner concept, an impedance tube sample (cylindrical sample, $39.5 \mathrm{~mm}$ diameter) and a B2A rectangular sample $(50 \mathrm{~mm} \times 150 \mathrm{~mm}$ ) have been manufactured by ATECA and Polyshape. Two types of liner are designed. The Medium Frequency (MF) liner is a classical SDOF with cavity full of melamine foam in order to enlarge the frequency domain of absorption. It is designed for middle range frequencies (over $1000 \mathrm{~Hz}$ ). The perforated plate is made of composite in order to comply with the weight constraints. Some pictures are shown in Figure 3.
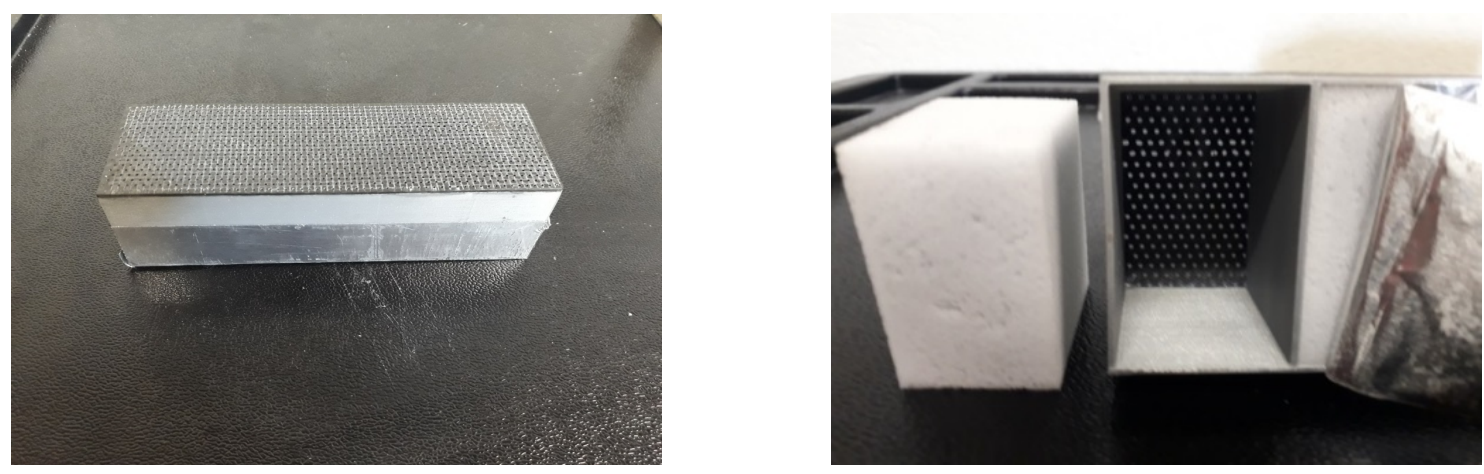

Figure 3. View of MF liner sample for B2A duct and view inside the liner

The Low Frequency (LF) liner concept is dedicated to low frequencies (below $1000 \mathrm{~Hz}$ ). It is a so-called LEONAR ("Long Elastic Open Neck Acoustic Resonator") concept. ${ }^{6}$ It is made of a perforated surface coupled with tubes of variable lengths inserted in a back cavity. Two tube lengths are chosen in order to enlarge the frequency range. These liners are considered as linear regarding the incident Sound Pressure Level (SPL). Figure 4 presents pictures of the liner sample. LF liners have been made by Polyshape, through an additive manufacturing process based on a resin.
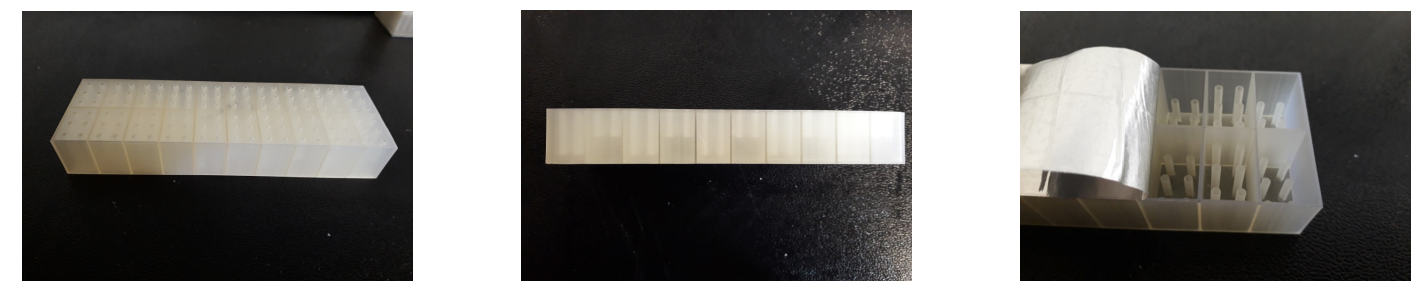

Figure 4. View of LF liner sample for B2A (left, middle and right)

\section{II.C. Description of the experimental setups}

\section{II.C.1. Impedance tube and ONERA B2A duct}

First, the acoustic impedance measurements are carried out in an impedance tube. It consists of a tube with a speaker at one end and a sample holder at the other end. Along the tube, 3 microphones are distributed for the measurement of sound pressure. The samples are cylindrical with a $39.5 \mathrm{~mm}$ diameter.

Then, the B2A flow duct has been used. The B2A aeroacoustic test bench at ONERA is made of a stainless steel tube of section $50 \mathrm{~mm} \times 50 \mathrm{~mm}$; its total length is about $4 \mathrm{~m}$. A $0.2 \mathrm{~m}$-long test section is equipped with two silica windows for optical access. The termination is equipped with a quasi-anechoic outlet, leading to an upstream reflection coefficient smaller than 0.2 for frequencies higher than $500 \mathrm{~Hz}$. A mean flow of bulk Mach number up to 0.3 can be provided. The flow temperature can be accurately regulated, from the ambient temperature up to $300^{\circ} \mathrm{C}$. All along the study, the flow temperature was imposed to be equal to $20^{\circ} \mathrm{C}$. In the test section, this flow shows fully developed turbulent boundary layers, with a turbulence rate of a few percent at the center of the test section. As shown in Fig. 5, flow propagates from left to right. Two acoustic drivers are mounted upstream of the test section, and are used to generate tones (usually multi-sine signal) at up to $140 \mathrm{~dB}$ over a frequency range of 0.3 to $3.5 \mathrm{kHz}$ (i.e., the no-flow cut-off frequency of the duct). When a multi-sine source is used, the OASPL within the duct is much higher 
(usually $10 \mathrm{~dB}$ more) than the SPL at each tone frequency. The surface of the test liner forms a $150 \mathrm{~mm}$-long portion of the lower wall of the flow duct. It should be noted that the liner spans all the duct width.

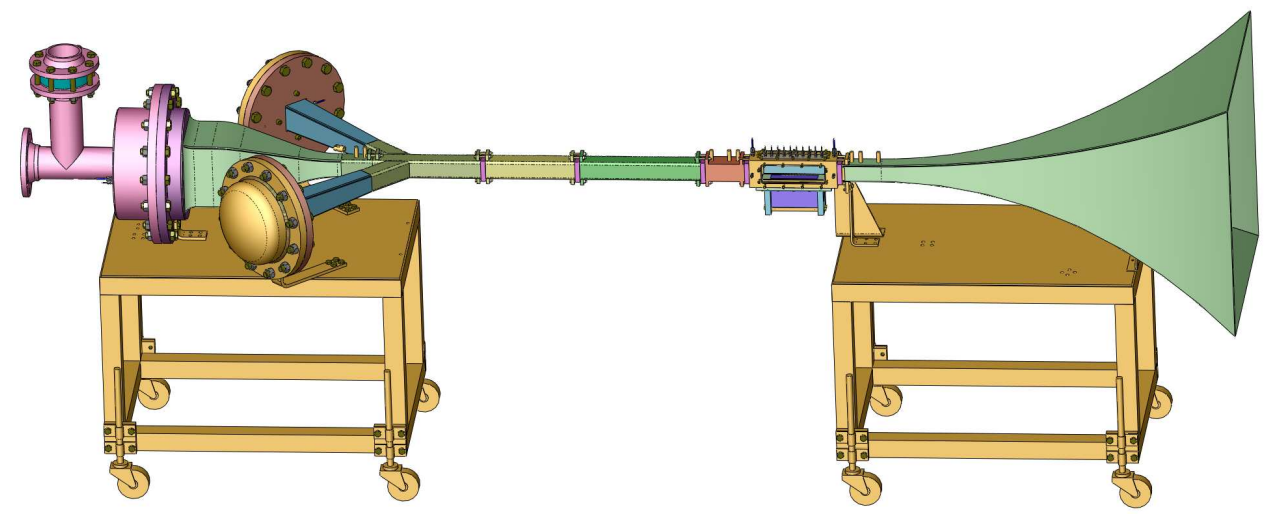

Figure 5. Sketch of B2A Aero-Thermo-Acoustic test bench.

Sixteen microphone locations are available in the upper wall (opposite the liner) to measure the acoustic pressure field from upstream of the liner leading edge to downstream of the liner trailing edge. Flush-mounted GRAS 40SC microphone probes are used (probe diameter of $1.2 \mathrm{~mm}$ ). Usually, only two upstream locations are considered to measure the amplitude of the incident acoustic plane waves. ${ }^{7}$ However, for the pressurebased impedance eduction method, one microphone probe is moved along the whole set of microphone locations. The cross-spectrum between the microphone and driver signal is acquired, which provides a phase reference for the pressure measurements.

\section{II.D. Instrumentation and impedance eduction methods}

\section{II.D.1. Two-microphone method for measuring the incident and reflected pressure waves}

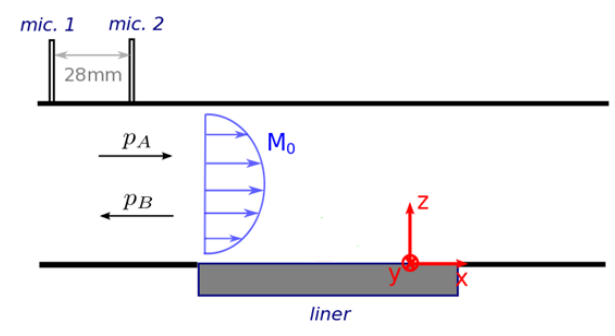

Figure 6. Sketch of the wave sorting procedure.

Both in the impedance tube and in the B2A tests, a two-microphone method is used to decompose the sound field in the duct into an incident and a reflected wave. ${ }^{7}$ This analysis is only performed below the first cut-off frequency of the duct, so that only plane waves are considered. In the impedance tube configuration, this method yields directly the reflection coefficient $\beta$ of the liner sample, and therefore its impedance $Z=\frac{1+\beta}{1-\beta}$ (made dimensionless by the impedance of air). This two-microphone method is also used in the B2A duct to assess the sound pressure level incident into the sample, i.e the sound pressure level of the downstream wave denoted by $A$ in the figure 6 , while the upstream reflected wave is denoted by $B$.

Two microphone probes GRAS 40SC are placed flush to the flow, at a distance $x_{1}$ and $x_{2}$ of the sample and therefore of the wall impedance discontinuity. We note $p_{1}$ and $p_{2}$ the sound pressure they measure. The spacing between the two probes is $2.8 \mathrm{~cm}$, which according to Boden \& $\mathrm{Abom}^{8}$ minimizes errors in the $[600,3450] \mathrm{Hz}$ frequency range. In order to also minimize measurement errors at lower frequencies, the measurements are repeated by switching the two microphones and an average of the obtained frequency response function is taken: this makes it possible to overcome the intrinsic phase shift between the microphones. Using the complex notation, and by introducing the angular frequency $\omega=2 \pi f$ of the considered frequency, 
the pressure field in the test section at a given position $x$ reads:

$$
p(x, t)=\operatorname{Re}\left(P(x) e^{i \omega t}\right)
$$

with $P(x)=A e^{-i k^{+} x}+B e^{i k^{-} x}$ where $k^{+}$and $k^{-}$are the wave numbers and $\mathrm{A}$ and $\mathrm{B}$ are the complex amplitudes of the forward and backward waves. The $\mathrm{x}$-axis is oriented from left to right and has its origin at the liner upstream location, i.e. at the position of the impedance discontinuity. For a uniform flow of Mach number $M$, we have:

$$
k^{ \pm}= \pm \frac{\omega / c}{1 \pm M}=\frac{ \pm k}{1 \pm M}
$$

with $k=\omega / c$. The two-microphone method allows the assessment of the sound pressure level $S_{A}$ of the downstream wave (i.e. $20 \log \frac{P_{A}^{r m s}}{2 \cdot 10^{-5}}$ with $P_{A}^{r m s}=\frac{|A|}{\sqrt{2}}$ ) from the sound pressure levels $S_{1}$ and $S_{2}$ measured at the microphones, by solving the following set of equations:

$$
\begin{aligned}
& A e^{-i \frac{k}{1+M} x}+B e^{i \frac{k}{1-M} x}=P_{1} \\
& A e^{-i \frac{k}{1+M} x}+B e^{i \frac{k}{1-M} x}=P_{2}
\end{aligned}
$$

which yields:

$$
\begin{aligned}
& A=\frac{P_{1} \exp \frac{i k x_{2}}{1-M}}{2 i \sin \frac{k \Delta x}{1-M^{2}} \exp \frac{i k M\left(x_{1}+x_{2}\right)}{1-M^{2}}}-\frac{P_{2} \exp \frac{i k x_{1}}{1-M}}{2 i \sin \frac{k \Delta x}{1-M^{2}} \exp \frac{i k M\left(x_{1}+x_{2}\right)}{1-M^{2}}}, \\
& B=\frac{P_{1} \exp \frac{-i k x_{2}}{1-M}}{2 i \sin \frac{k \Delta x}{1-M^{2}} \exp \frac{i k M\left(x_{1}+x_{2}\right)}{1-M^{2}}}-\frac{P_{2} \exp \frac{-i k x_{1}}{1-M}}{2 i \sin \frac{k \Delta x}{1-M^{2}} \exp \frac{i k M\left(x_{1}+x_{2}\right)}{1-M^{2}}} .
\end{aligned}
$$

The following expression can then be derived:

$$
\begin{aligned}
|A|^{2} & =\frac{\left|P_{1}\right|^{2}}{\left|2 \sin \frac{k \Delta x}{1-M^{2}}\right|^{2}} 1-2 \operatorname{Re}\left(H_{21} e^{-i \frac{k \Delta x}{1-M}}\right)+\left|H_{21}\right|^{2} \\
|B|^{2} & =\frac{\left|P_{1}\right|^{2}}{\left|2 \sin \frac{k \Delta x}{1-M^{2}}\right|^{2}} 1-2 \operatorname{Re}\left(H_{21} e^{i \frac{k \Delta x}{1+M}}\right)+\left|H_{21}\right|^{2}
\end{aligned}
$$

with $\Delta x=x_{2}-x_{1}$ and $H_{21}=\left|H_{21}\right| e^{i \phi_{21}}=P_{2} / P_{1}$ the frequency response function between the microphones. The sound pressure levels of the downstream and upstream waves read thus (in decibels, $S_{B}$ being defined from $|B|$ in the same way as $S_{A}$ has been defined from $\left.|A|\right)$ :

$$
\begin{aligned}
& S_{A}=S_{1}-20 \log \left(2\left|\sin \frac{k \Delta x}{1-M^{2}}\right|\right)+10 \log \left(1+2\left|H_{21}\right| \cos \left(\phi_{21}-\frac{k \Delta x}{1-M}\right)+\left|H_{21}\right|^{2}\right) \\
& S_{B}=S_{1}-20 \log \left(2\left|\sin \frac{k \Delta x}{1-M^{2}}\right|\right)+10 \log \left(1+2\left|H_{21}\right| \cos \left(\phi_{21}-\frac{k \Delta x}{1+M}\right)+\left|H_{21}\right|^{2}\right)
\end{aligned}
$$

In the present study, $M$ is chosen equal to $M_{b}$, the bulk flow Mach number.

\section{II.D.2. Non-intrusive measurement of the acoustic velocity field}

The B2A duct is equipped with a Laser Doppler Anemometry (LDA) system, in order to measure the streamwise and vertical velocity components $u$ and $w$ of the flow. As this measurement technique is timeresolved, both the mean and fluctuating parts of the velocity can be measured. Especially, the acoustic velocity field can be obtained with a specific post-treatment technique. The acoustic velocity is defined here as the component of the signal that is correlated with the loudspeaker signal. It can be educed from the extraneous noise (mainly due to the turbulent flow) by a technique similar to the three-microphone signal enhancement technique. ${ }^{7,9}$ It consists in calculating the cross-spectral density function $G_{U_{i}, l s}$ between the 
velocity signal $U_{i}$ and the loudspeaker signal $l s\left(\operatorname{see}^{10-12}\right)$. The auto-spectral density function of the acoustic velocity reads:

$$
G_{u_{i}}=\frac{\left|G_{U_{i}, l s}\right|^{2}}{G_{l s}}
$$

where $G_{l s}$ is the auto-spectral density function of the loudspeaker signal. The acoustic velocity in the frequency domain is then given by

$$
u_{i}=\sqrt{G_{u_{i}}} \exp \left[\mathrm{j} \Phi\left(U_{i} / l s\right)\right],
$$

where the phase of the acoustic velocity, referenced by the loudspeaker, is defined as

$$
\Phi\left(U_{i} / l s\right)=\arctan \frac{\operatorname{Im}\left(G_{U_{i}, l s}\right)}{\operatorname{Re}\left(G_{U_{i}, l s}\right)} .
$$

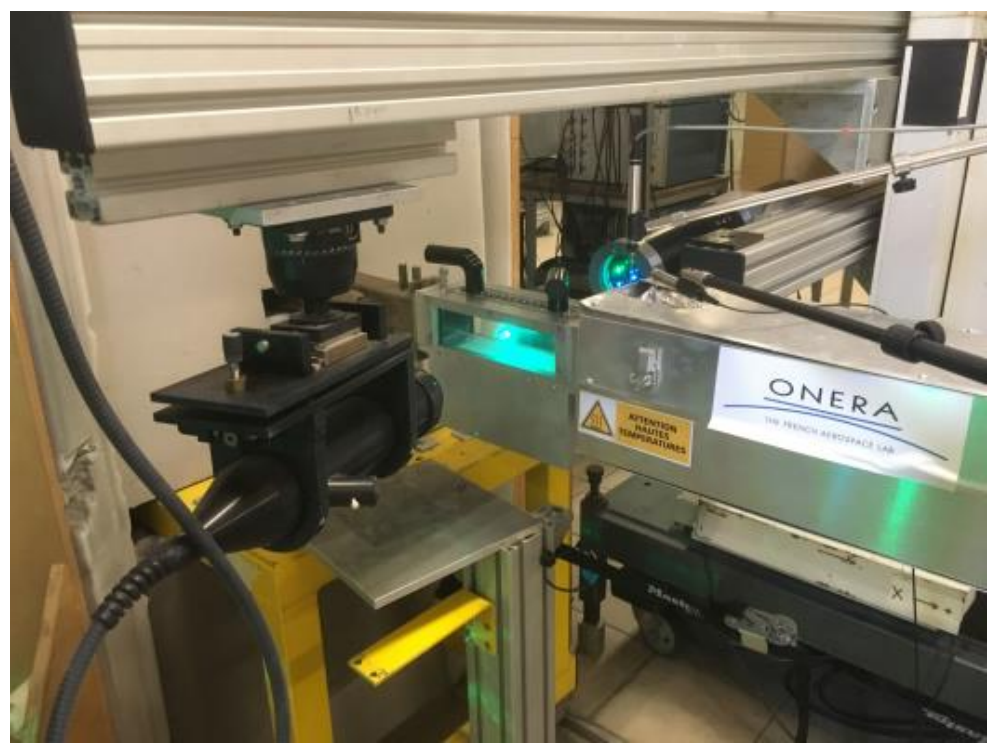

Figure 7. On going LDA measurement on B2A

\section{II.D.3. Impedance Eduction Methodology}

In the impedance tube configuration, the impedance is directly derived from the measured reflection coefficient, as explained previously. In the B2A configuration, a more complex methodology has to be used : an impedance eduction method, which is based on the two-dimensional time-harmonic linearized Euler equations (LEE). The underlying model is briefly summarized here for convenience, but all details can be found in Primus et al. ${ }^{13}$

The linearized Euler equations, written in non-conservative form, with an $e^{j \omega t}$ time dependence, assuming homentropic flow is:

$$
\mathbf{L} \varphi=0
$$

where

$$
\mathbf{L} \boldsymbol{\varphi}=\mathrm{j} \omega \boldsymbol{\varphi}+\boldsymbol{A}_{x} \frac{\partial \boldsymbol{\varphi}}{\partial x}+\boldsymbol{A}_{z} \frac{\partial \boldsymbol{\varphi}}{\partial z}+\boldsymbol{B} \boldsymbol{\varphi}
$$

and

$$
\boldsymbol{A}_{x}=\left(\begin{array}{ccc}
U & 0 & c_{0} \\
0 & U & 0 \\
c_{0} & 0 & U
\end{array}\right), \boldsymbol{A}_{7}=\left(\begin{array}{ccc}
W & 0 & 0 \\
0 & W & c_{0} \\
0 & c_{0} & W
\end{array}\right), \boldsymbol{B}=\left(\begin{array}{ccc}
\partial_{x} U & \partial_{z} U & 0 \\
\partial_{x} W & -\partial_{x} U & 0 \\
0 & 0 & 0
\end{array}\right)
$$


Components of vector $\varphi=\left(u, w, \frac{p}{\rho_{0} c_{0}}\right)^{\top}$, where $\rho_{0}$ is the density of the mean flow, represent the acoustic perturbations around the sheared mean flow of axial and transverse velocities $U$ and $W$, respectively. The flow being assumed homentropic, the energy equation is replaced by the state equation $p=c_{0}^{2} \rho_{0}$, with $\rho_{0}$ the acoustic density.

As we consider a sheared mean flow profile, the impedance boundary condition on the lined wall is the standard one

$$
p= \pm \rho_{0} c_{0} Z w
$$

where the sign is chosen depending on whether the upper or lower wall is considered. To avoid any singularities in the rigid wall case, the boundary condition (16) is expressed as a function of the normal incidence reflection coefficient $\beta=(Z-1) /(Z+1)$, leading to the following boundary condition matrix:

$$
\boldsymbol{M}_{\beta}=\frac{1}{2}\left(\begin{array}{cc}
(\beta+1) \boldsymbol{n} \otimes \boldsymbol{n} & (\beta-1) \boldsymbol{n} \\
-(\beta+1) \boldsymbol{n}^{\top} & (1-\beta)
\end{array}\right)=0
$$

where $\boldsymbol{n}$ is the local normal vector and $\otimes$ the tensor product. On the rigid walls the reflection coefficient $\beta$ is then taken as 1 .

At the source plane, the state vector $\varphi$ is taken to be equal to $\mathcal{C} \varphi_{0}$, where $\varphi_{0}$ is the plane-wave solution in a rigid duct with uniform flow Mach number $M_{0}$, corresponding to the bulk Mach number of the actual shear flow $U(z)$. As this plane-wave solution is chosen with an arbitrary amplitude taken to be equal to 1 , the complex coefficient $\mathcal{C}$ stands for the source amplitude. $\mathcal{C}$ can be either set to the measured value of the acoustic pressure at the source plane, when available, or included into the eduction process to reproduce at best the measured acoustic field. At the exit plane, either a non-reflecting or an exit impedance boundary condition can be enforced. The first option is only used when the data suggests that this is proper, i.e. when no standing-wave can be observed in the downstream hard wall section. As the LDA measurement system does not extend to the exit plane, this information can only be provided by microphone measurements downstream of the lined section. As these additional measurements are not systematically performed, the exit impedance boundary condition is commonly used. This will be the case for all the computations shown in this paper. As a consequence, during the search procedure, both $\mathcal{C}$ and $Z_{t}$ exit impedance are used as design variables, in addition to $Z$.

A discontinuous Galerkin (DG) scheme is chosen to solve Eq. (14) and the associated boundary conditions. In this method, discontinuities are allowed at the interface between two elements. An example of how such a method can handle discontinuities at hard-soft wall interfaces is found elsewhere. ${ }^{14} \mathrm{~A}$ triangular mesh of Lagrange $\mathbb{P}_{1}$ elements is used. To ensure the connection between elements and to apply the boundary conditions, a numerical flux is defined. An upwind numerical flux is used to ensure connection between interior cells, while a centered flux is chosen at the boundaries (see previous papers ${ }^{13,15}$ for more details).

The inverse problem is defined by the minimization of the following objective function:

$$
\mathcal{J}_{\text {red }}=\frac{\int_{\Omega_{\text {obs }}}\left\{\left.\boldsymbol{\varphi}\right|_{\mathrm{DG}}-\left.\boldsymbol{\varphi}\right|_{\text {Meas }}\right\}^{\top}\left\{\left.\bar{\varphi}\right|_{\mathrm{DG}}-\left.\bar{\varphi}\right|_{\text {Meas }}\right\} \mathrm{d} x \mathrm{~d} z}{\int_{\Omega_{\text {obs }}}\left\|\left.\boldsymbol{\varphi}\right|_{\text {Meas }}\right\|^{2} \mathrm{~d} x \mathrm{~d} z}
$$

where $\Omega_{o b s}$ is the observation region.

Two different configurations are considered. When the impedance eduction method is based on acoustic pressure measurements on the wall opposite the test liner, $\Omega_{o b s}$ is made of the discrete set of microphone locations at the wall, and only the pressure component of the state vector is considered in the objective function (18). When the impedance eduction method is based on LDA measurements above the test liner (see Fig. 8), $\Omega_{o b s}$ is a rectangular $(x, z)$ region and the $(u, w)$ velocity components of the state vector are considered in function (18).

A BFGS-B (Broyden, Fletcher, Goldfarb, Shanno for bounded variables) algorithm is used to solve the optimization problem. ${ }^{16}$ The analytical expression of the gradient of $\mathcal{J}$ with respect to each of the search parameters $Z, Z_{t}, \mathcal{C}$ is obtained via an adjoint method, as explained in Primus et al.. ${ }^{13}$

$$
\begin{aligned}
\frac{\partial \mathcal{J}}{\partial Z} & =-\left\langle\frac{\partial \boldsymbol{M}_{\beta}}{\partial \beta} \frac{\partial \beta}{\partial Z} \boldsymbol{\varphi}, \boldsymbol{\varphi}^{*}\right\rangle_{\Gamma_{l}} \\
\frac{\partial \mathcal{J}}{\partial Z_{t}} & =-\left\langle\frac{\partial \boldsymbol{M}_{\beta_{t}}}{\partial \beta_{t}} \frac{\partial \beta_{t}}{\partial Z_{t}} \boldsymbol{\varphi}, \boldsymbol{\varphi}^{*}\right\rangle_{\Gamma_{t}} \\
\frac{\partial \mathcal{J}}{\partial \mathcal{C}} & =\left\langle\boldsymbol{A}^{i} n_{i}^{-} \boldsymbol{\varphi}_{0}, \boldsymbol{\varphi}^{*}\right\rangle_{\Gamma_{s}}
\end{aligned}
$$




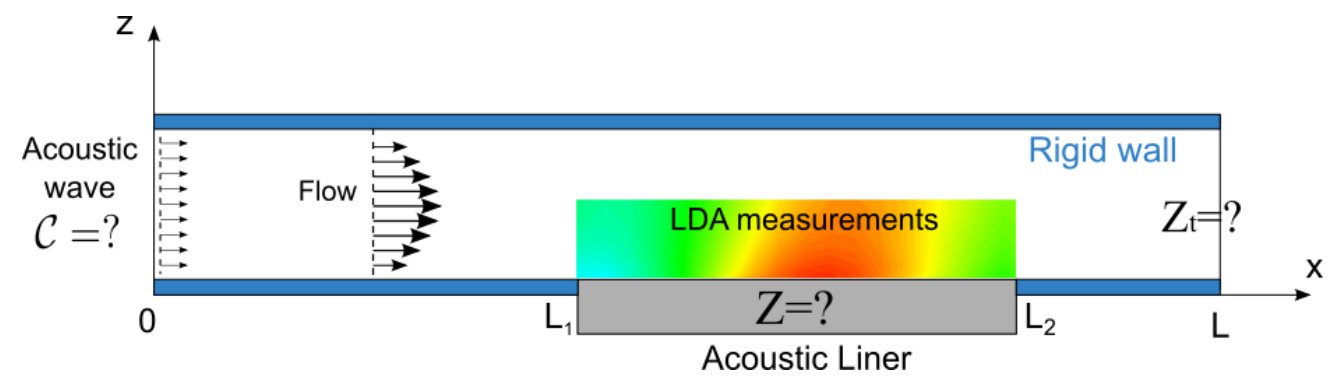

Figure 8. Impedance eduction from LDA measurements

where $\boldsymbol{\varphi}^{*}$ is the solution of the adjoint problem and $\boldsymbol{A}^{i} n_{i}^{-}$is the matrix associated to the negative eigenvalues of the matrix $\boldsymbol{A}^{i} n_{i}=\boldsymbol{A}_{x} \boldsymbol{n}_{x}+\boldsymbol{A}_{z} \boldsymbol{n}_{z} . \Gamma_{l}, \Gamma_{t}$ and $\Gamma_{s}$ are the liner surface, the exit plane and the source plane, respectively.

\section{II.E. Pressure drop measurement}

Static pressure measurements are performed with a SVMtec pressure multisensor. The SVMtec pressure multisensor is used with a $1.25 \mathrm{kPa}$ range with an accuracy of $+/-0.1 \%$ FSS. It measures the static pressure at the wall with the first pressure tap taken as a reference. This type of pneumatic configuration enables to measure directly the pressure drop in the B2A test section. The measurements give the static pressure difference between the current pressure tap and the first pressure tap. The frequency sampling is $f=10 \mathrm{~Hz}$. These acquisitions are taken during $40 \mathrm{~s}$. The mean part of these acquisitions is considered in the following.

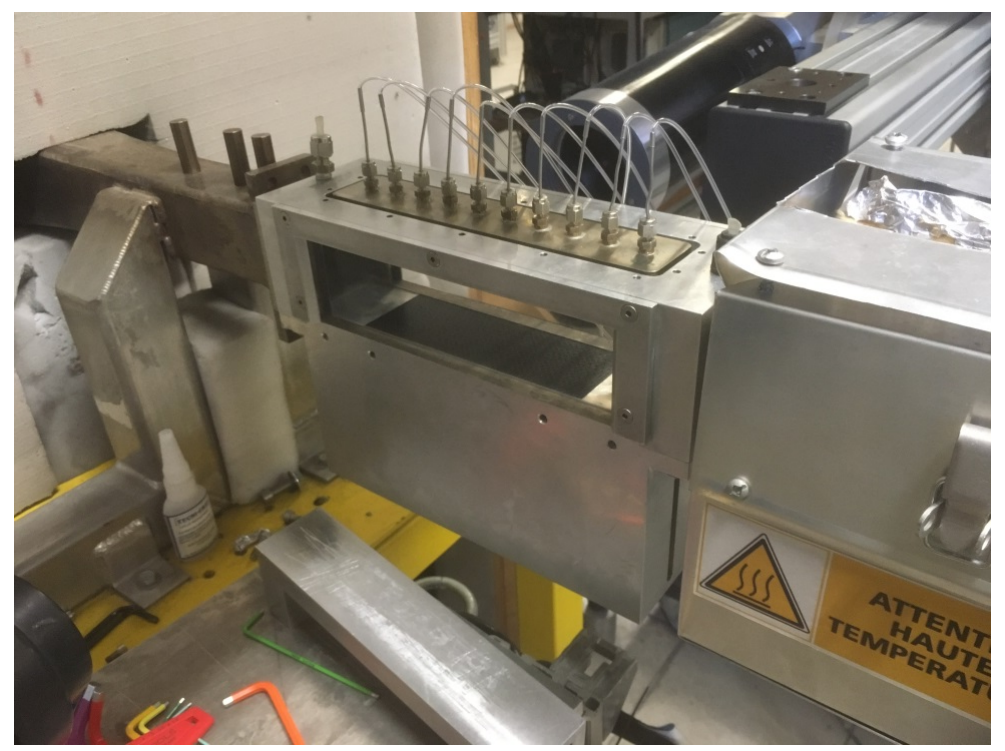

Figure 9. Pressure taps set up for B2A

For this investigation, the relative drag of each configuration will be determined by measuring differences in the static pressure drop along the duct wall opposite of the liner sample. ${ }^{17}$ The static pressure drop measurements enables a balance of the momentum within the test section. With the static drop measurement and the flow parameter, the duct resistance factor $\lambda$ can be computed ${ }^{18}$ :

$$
\lambda=-\frac{d p_{0}}{d x} \frac{h}{q}
$$

using the duct height $h$ as the hydraulic diameter and the incompressible form of $q$ :

$$
q=\frac{1}{2} \rho_{0} V_{b}^{2}
$$


where $V_{b}$ is the bulk velocity. From the static pressure drop measurements, we can therefore assess of an apparent friction velocity of the test section, through the following equation: ${ }^{19}$

$$
v^{*}=\sqrt{-\frac{h}{4 \rho_{0}} \frac{\mathrm{d} p_{0}}{\mathrm{~d} x}} .
$$

As a matter of comparison, we compared the obtained result to Prandtl's friction law for smooth pipes that reads :

$$
\frac{1}{\sqrt{f_{D}}}=2.0 \log _{10}\left(R_{D_{h}} \sqrt{f_{D}}\right)-0.8
$$

where $f_{D}=4 C_{f}=8\left(v^{*} / U_{b}\right)^{2}$ is the average (Darcy) friction factor and $R_{D_{h}}$ is the Reynolds number based on the hydraulic diameter $D_{h}$. For square ducts, taking $D_{h}=2 h$ leads to satisfactory estimates of $v^{*}$ using Eq. $24 .{ }^{20}$

\section{Results and discussion}

\section{III.A. Friction measurement}

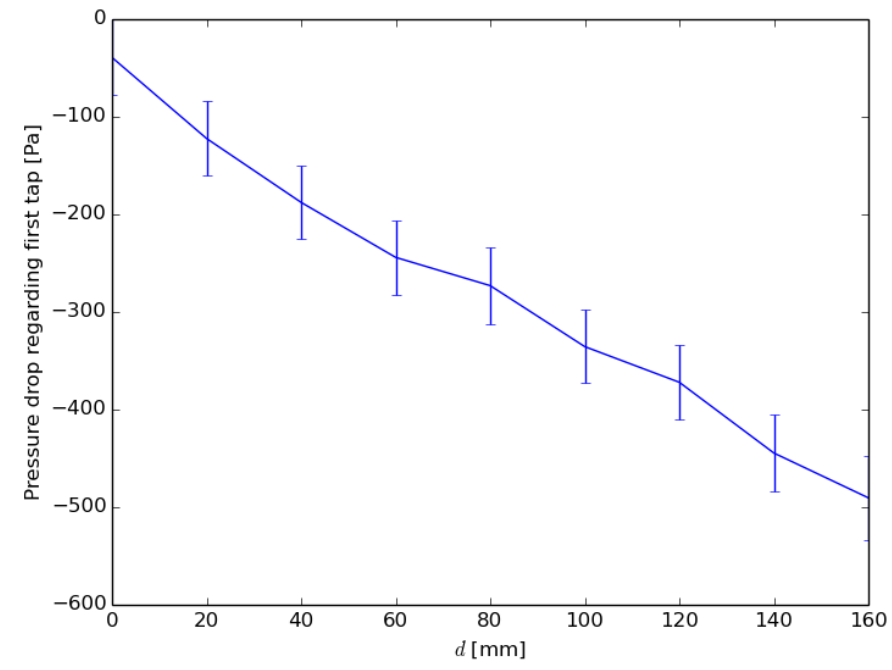

Figure 10. Pressure drop along the wall opposite the liner in the B2A duct for the MF Sample.

Figure 10 presents an example of pressure drop along the MF liner in the B2A duct for a typical Mach number of 0.3 . The results are presented with a confidence interval at $95 \%$ derived from a statistical analysis of the pressure results.

\begin{tabular}{|c|c|c|c|}
\hline Sample & $v^{*}(m / s)$ for $M_{b}=0.15$ & $v^{*}(m / s)$ for $M_{b}=0.2$ & $v^{*}(m / s)$ for $M_{b}=0.3$ \\
\hline Eq 24 results & 2.25 & 2.92 & 4.15 \\
\hline LF & 2.39 & 3.18 & 5.0 \\
\hline MFL & 2.66 & - & 5.3 \\
\hline
\end{tabular}

Table 1. Friction velocity results

Tab 1 sums up the different friction velocity results. In order to reduce the overall mass of the liner so that the industrial requirements can be fulfilled, pre-peg composites are used for the perforated sheet the MF concept while the LF liner is made of resin. The friction velocity is a driving parameter regarding the effect of the flow on the acoustic resistance of the liner. The liner surface has to be as smooth as possible in order to reduce the acoustic resistance increase due to the grazing flow. 


\section{III.B. Impedance results}

III.B.1. MFL liner

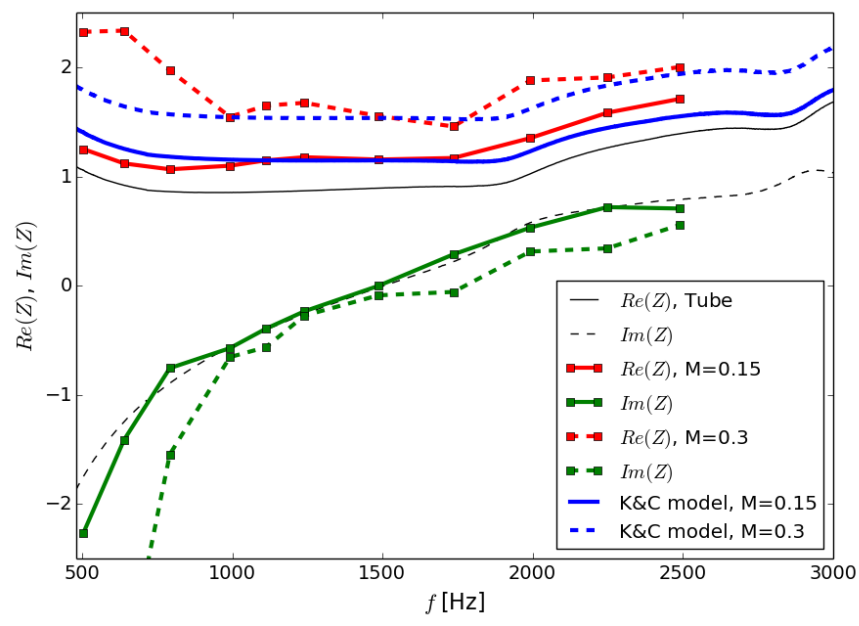

Figure 11. MFL normalized impedance result for several Mach number

The eduction method is applied to B2A measurements in order to assess the impedance of the liner under a grazing flow. Figure 11 shows a comparison between the impedance tube result (black line) of the MF liner concept and the same liner concept under grazing flow $\left(M_{b}=0.15\right.$ and $\left.M_{b}=0.3\right)$. The flow tends to increase the resistance of the liner. The effect is assumed to be neglectible on the reactance, at least at $M_{b}=0.15$. Kirby \& Cummings ${ }^{21}$ proposed an impedance model for a perforated sheet under a grazing flow. It has to take into account also the friction velocity $v^{*}$ and the sound velocity $c_{0}$ of the grazing flow. The impedance of the perforated sheet reads

$$
Z_{\text {perforated sheet }}=R+i X
$$

with

$$
\begin{array}{r}
R=\frac{\sqrt{8 \nu \omega}}{P O A \cdot c_{0}} \frac{\delta}{\phi}+\frac{\left(26.16\left(\frac{\delta}{\phi}\right)^{-0.169}-20\right) v^{*}}{P O A \cdot c_{0}}-\frac{4.055 f \phi}{P O A \cdot c_{0}} \\
X=\frac{\omega}{P O A \cdot c_{0}}\left(\delta+\epsilon \frac{8 \phi}{3 \pi}\right) \\
\epsilon=\min \left[1,\left(1+0.6 \frac{\delta}{\phi}\right) \exp -\frac{\frac{v^{*}}{f \delta}-0.18 \frac{\phi}{\delta}}{1.8+\frac{\delta}{\phi}}-0.6 \frac{\delta}{\phi}\right]
\end{array}
$$

Thus the model can be used to predict the resistance of the liner for several values of the friction velocity.

On Figure 11, the Kirby \& Cummings model is applied considering the friction velocity derived from the pressure drop measurements. The model is applied only on the resistance. The effect on the reactance can be neglected. There is a very good agreement with the B2A impedance results. The Kirby \& Cummings model is then convenient to evaluate the real resistance value in the jet pump flow configurations if the friction velocity can be evaluated, which can be provided by a RANS simulation. The efficiency of the liner could then be evaluated from a realistic predicted impedance that take into account the effect of the grazing flow.

\section{III.B.2. LF liner}

Figures 13 and 12 emphasize the effect of the sound pressure level on the normal absorption coefficient and on the impedance value of the LF liner without grazing flow (impedance tube). This LF liner technology enables to have a linear behavior regarding the sound pressure level. 


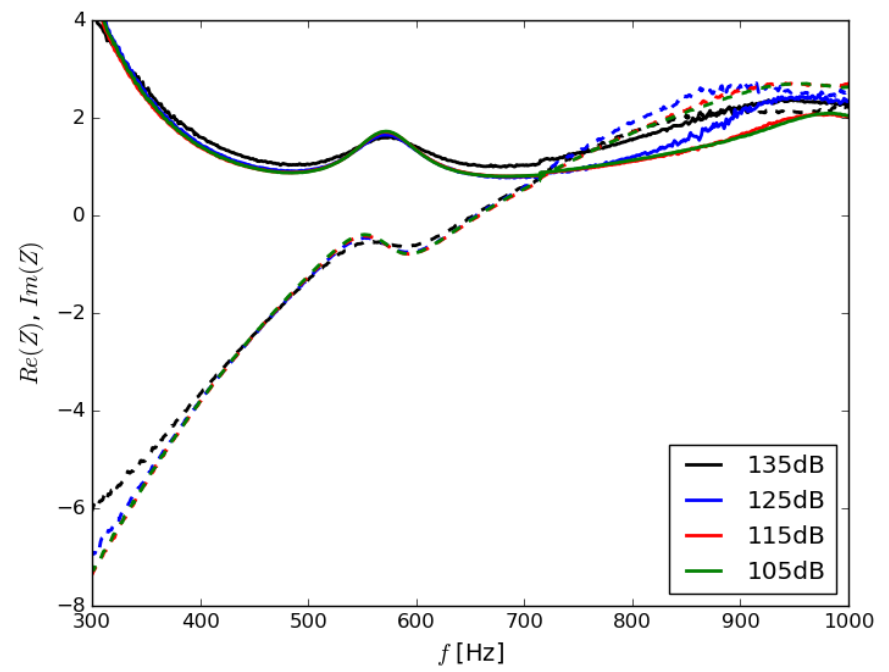

Figure 12. Effect of OASPL on the impedance on the LF liner in the impedance tube

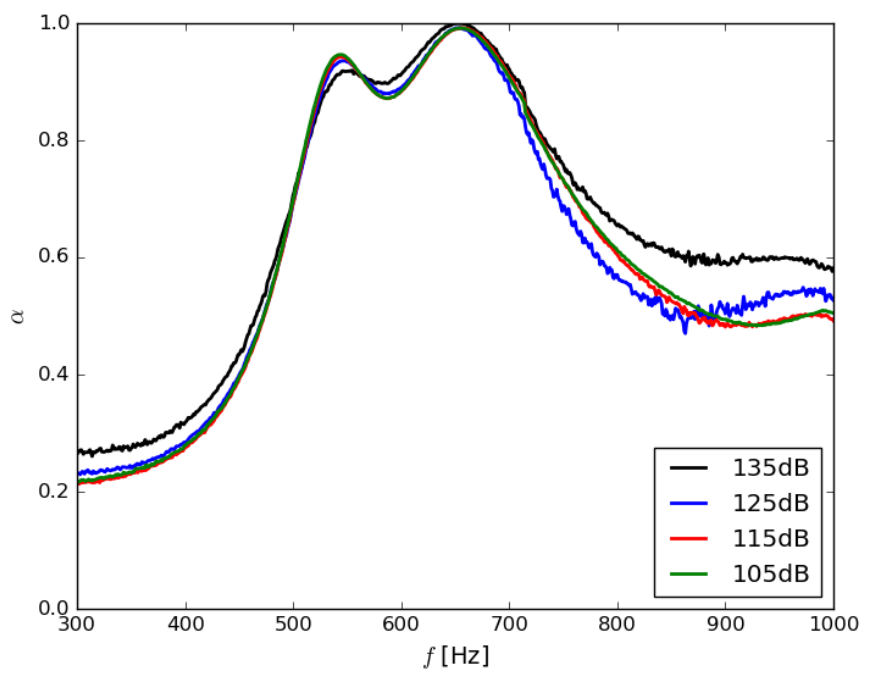

Figure 13. Effect of OASPL on the normal absorption coefficient on the LF liner in the impedance tube 


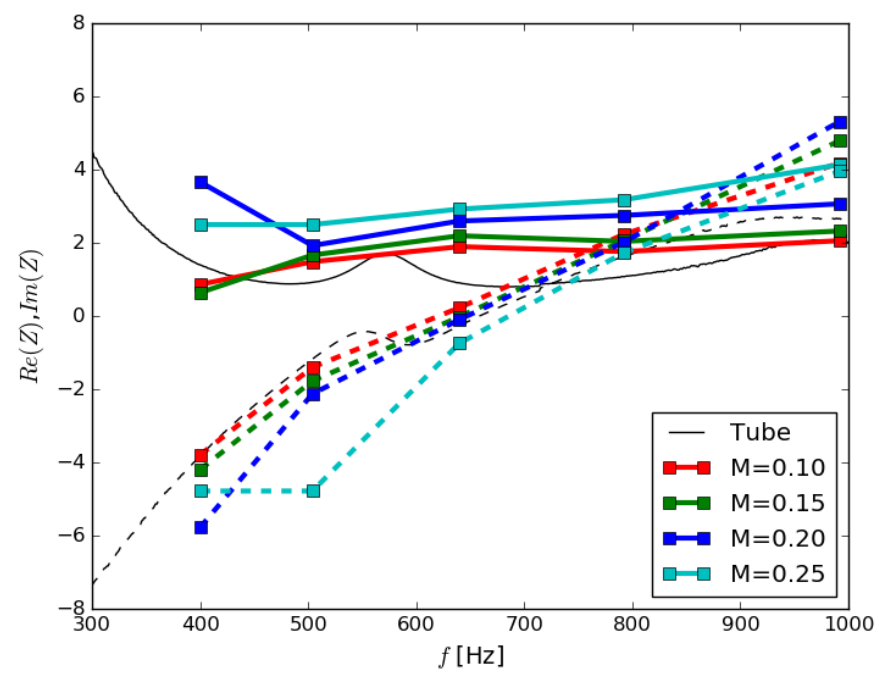

Figure 14. LF normalized impedance result for several Mach number

Figure 14 presents the results for LF concept under grazing flow. Note that for the LEONAR concept, no formula is available yet to take into account the flow effect on the impedance. Therefore, several Mach number have been tested in order to assess the effect of the flow on the resistance. Similarly to classical SDOF with perforated sheet, the flow seems to increase the resistance of the liner. In that particular case, Kirby \& Cummings model decreases the resistance value. Guess ${ }^{22}$ proposed a correction $\theta_{\text {Guess }}$ for the effect of grazing flow on the resistance:

$$
\theta_{\text {Guess }}=\frac{1-P O A^{2}}{P O A} 0.3 M_{b}
$$

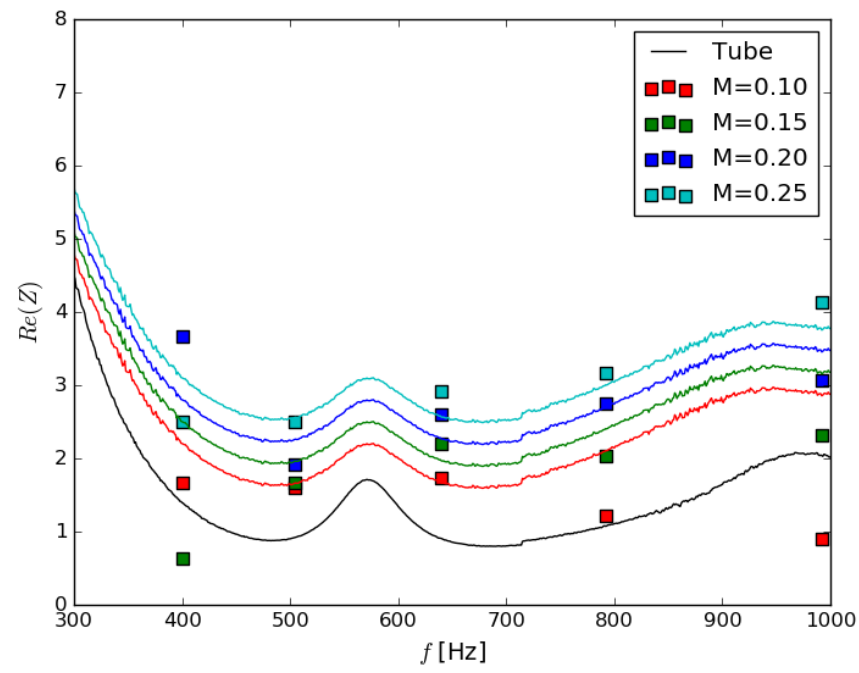

Figure 15. LF normalized resistance experimental results (markers) compared to the normalized resistance from the impedance tube corrected by Guess term

Figure 15 shows the effect of Guess grazing flow effect correction applied to the impedance resistance tube result: the agreement with experiments is fairly good around the resonnance frequencies (when the reactance is null, around $500 \mathrm{~Hz}$ to $650 \mathrm{~Hz})$. 


\section{Conclusion}

This paper presented the experimental characterization of two innovative liner concepts under grazing flow conditions. The first concept is a classical SDOF (perforated sheet over a cavity) with acoustic foam inside the resonnant cavity. The effect of the grazing flow on the impedance is similar to classical SDOF and can be undertaken with classical models. The second concept is a LEONAR liner which is linear regarding the sound pressure level but effects due to the grazing flow on the resistance have been highlighted. Even if no model is available to reproduce the effect of the grazing flow on the resistance, using Guess correction for grazing flow effect is a fair solution. The proposed liner are fully characterized and the impedance can be forecasted in any grazing flow conditions. Acoustics simulations can thus be performed in order to evaluate the efficiency of such liners in industrial configurations (presently for the jet pump), which will be done during the last year of the IDEAS project.

\section{Acknowledgments}

This project has received funding from the Clean Sky 2 Joint Undertaking under the European Union's Horizon 2020 research and innovation programme under grant agreement No 716499. ATECA and PolyShape are gratefully acknowledged for the manufacturing of the liner samples.

\section{References}

\footnotetext{
${ }^{1}$ Law, T., Dowling, A., and Corral, R., "Optimisation of axially segmented liners for aeroengine broadband noise," $J$. Sound Vib., Vol. 329, 2010, pp. 4367-4379.

${ }^{2}$ Mustafi, P., Astley, J., and Sugimoto, R., "A computational study of the effects of liner damage on zero-splice turbofan intake liners," 18th AIAA/CEAS Aeroacoustics Conference (33rd AIAA Aeroacoustics Conference), Colorado Springs, Colorado, USA, June 2012, AIAA-2012-2152.

${ }^{3}$ Tester, B., "The propagation and attenuation of sound in lined ducts containing uniform or "plug" flow," Journal of Sound and Vibration, Vol. 28, No. 2, May 1973, pp. 151-203.

${ }^{4}$ Malmary, C., Etude Théorique et Expérimentale de L'impédance Acoustique de Matériaux En Présence D'un Ecoulement D'air Tangentiel, Ph.D. thesis, Université du Maine, 2000.

${ }^{5}$ Piot, E., Brazier, J.-P., Simon, F., Fascio, V., Peyret, C., and Ingenito, J., "Design, manufacturing and demonstration of acoustic liners for air conditioning systems," American Institute of Aeronautics and Astronautics, May 2016.

${ }^{6}$ F.Simon, "Long Elastic Open Neck Acoustic Resonator for low frequency absorption," , No. 421, 2018.

${ }^{7}$ Chung, J. Y. and Blaser, D. A., "Transfer function method of measuring in-duct acoustic properties. I. Theory," J. Acoust. Soc. Am., Vol. 68, No. 3, 1980, pp. 907-913.

${ }^{8}$ Boden, H. and Abom, M., "Influence of errors on the two microphones method for measuring acoustics properties in ducts," J. Acoust. Soc. Am., Vol. 79, No. 2, 1986, pp. 541-549.

${ }^{9}$ Nance, D. and Ahuja, K. K., "Limitations of the three-microphone signal enhancement technique," Proceedings of the 45th AIAA Aerospace Sciences Conference, AIAA-2007-441, Reno, Nevada, January 2007.

${ }^{10}$ Minotti, A., Simon, F., and Gantié, F., "Characterization of an acoustic liner by means of Laser Doppler Velocimetry in a subsonic flow," Aerospace Science and Technology, Vol. 12, No. 5, 2008, pp. 398-407.

${ }^{11}$ Lavieille, M., Simon, F., and Micheli, F., "Measurement of acoustic quantity fields in duct flow by Laser Doppler Velocimetry." Proceedings of the 12th AIAA/CEAS Aeroacoustics Conference, AIAA-2006-2550, Cambridge, Massachusetts, May 8-10 2006.

${ }^{12}$ Leon, O., Piot, E., Sebbane, D., and Simon, F., "Measurement of acoustic velocity components in a turbulent flow using LDV and high-repetition-rate PIV," Experiments in fluids, 2017.

${ }^{13}$ Primus, J., Piot, E., and Simon, F., "An adjoint-based method for liner impedance eduction: Validation and numerical investigation," J. Sound Vib., Vol. 332, No. 1, January 2013, pp. 58-75.

${ }^{14}$ Pascal, L., Piot, E., and Casalis, G., "Discontinuous Galerkin Method for acoustic modes computation in lined ducts," Proceedings of the 18th AIAA/CEAS Aeroacoustics conference, AIAA 2012-2153, Colorado Springs, Colorado, USA, 4-6 June 2012.

${ }^{15}$ Piot, E., Primus, J., and Simon, F., "Liner impedance eduction technique based on velocity fields," Proceedings of the 18th AIAA/CEAS Aeroacoustics Conference, AIAA 2012-2198, Colorado Springs, Colorado, USA, 4-6 June 2012.

${ }^{16}$ Byrd, R. H., Nocedal, J., and Schnabel, R. B., "A limited memory algorithm for bound constrained optimization," SIAM Journal on Scientific and Statistical Computing, Vol. 16, 1995, pp. 1190-1208.

${ }^{17}$ Howerton, B. M. and Jones, M. G., "Acoustic Liner Drag: A Parametric Study of Conventional Configurations," 21st AIAA/CEAS Aeroacoustics Conference, 2015, AIAA 2015-2230.

${ }^{18}$ J., N., "Laws of Flow in Rough Pipes," NASA TM-1292, Nov. 1950.

${ }^{19}$ Schlichting, H., Boundary layer theory, McGraw-Hill classic textbook reissue, 1979.

${ }^{20}$ Pirozzoli, S., Modesti, D., Orlandi, P., and Grasso, F., "Turbulence and secondary motions in square duct flow," Journal of Fluid Mechanics, Vol. 840, 2018, pp. 631-655.
} 
${ }^{21}$ Kirby, R. and Cummings, A., "The impedance of perforated plates subjected to grazing gas flow and backed by porous media," Journal of Sound and Vibration, Vol. 217, 1998, pp. 619-636.

${ }^{22}$ A.W.Guess, "Calculation of perforated plate liner parameters from specified acoustic resistance and reactance," Journal of Sound and Vibration, Vol. 40, No. 1, 1975, pp. 119 - 137. 